\title{
Impaired nasal breathing may prevent the beneficial effect of weight loss in the treatment of OSA*
}

\author{
Henry Blomster ${ }^{1}$, Tatu Kemppainen ${ }^{1}$, Jura Numminen ${ }^{2}$, Pirkko Ruoppi ${ }^{1}$, \\ Johanna Sahlman ${ }^{1}$, Markku Peltonen ${ }^{3}$, Juha Seppä ${ }^{1}$ and Henri Tuomilehto ${ }^{1}$
}

1 Departments of Otorhinolaryngology, Kuopio University Hospital, and Institute of Clinical Medicine, University of Eastern Finland

2 Department of Otorhinolaryngology, Tampere University Hospital, and University of Tampere, Finland

3 Chronic Disease Epidemiology and Prevention Unit, National Institute for Health and Welfare, Helsinki, Finland

SUMMARY Background: Weight loss is considered an effective treatment for obstructive sleep apnoea (OSA) in overweight patients. Some patients, however, do not benefit from weight loss. It has been postulated that nasal obstruction may act as an independent risk factor for OSA.

Objective: The aim of our study was to evaluate whether impaired nasal airflow might explain the missing effect of weight reduction on OSA.

Methodology: Fifty-two overweight adult patients with mild OSA were recruited. After the 12-month lifestyle intervention, all patients who achieved $\geq 5 \%$ weight loss were divided into two groups based on whether they still had OSA or not. Change in nasal resistance measured by rhinomanometer and AHI were the main outcome variables.

Results: A total of $26 / 52$ patients achieved 5\% weight reduction. Of those 26 patients, 16 were objectively cured from OSA and 10 patients did not benefit from weight loss. Nasal resistance reduced significantly more in patients who had been cured from OSA. Smoking had a negative impact on both nasal resistance and improvement of AHI.

Conclusions: Impaired nasal breathing and smoking may prevent the beneficial effects of weight reduction in the treatment of OSA.

Key words: obstructive sleep apnoea; nasal resistance; weight reduction; lifestyle intervention; cigarette smoking.

\section{INTRODUCTION}

Obstructive sleep apnoea (OSA) is a major health burden. OSA is found to be associated with increased morbidity and mortality ${ }^{(1,2)}$. It has been estimated that OSA affects $2-4 \%$ of men and $1-2 \%$ of women, mainly working aged individuals. Obesity is considered to be the most important risk factor for OSA ${ }^{(3)}$. There are other predispositions for OSA in adults e.g. male sex, craniofacial and upper airway abnormalities ${ }^{(1)}$. Nasal obstruction may also act as an independent risk factor for OSA ${ }^{(4)}$. It has been claimed that there is a correlation between obesity and nasal blockage ${ }^{(5)}$. However, our previous study observed that even an extensive weight loss did not invariably lead to beneficial changes in nasal breathing ${ }^{(6)}$.

Weight reduction has been reported to be beneficial in most overweight patients with OSA ${ }^{(7-9)}$, but in some patients the apnoea hypopnoea index (AHI) does not seem to improve even after a successful weight loss. The present study has evaluated whether impaired nasal airflow might explain part of the miss- ing effect of weight reduction on OSA in these patients. The hypothesis was that patients who had successful weight reduction but no improvement in OSA would display increased nasal resistance in the supine position.

The main outcome measures were total inspiratory nasal resistance in rhinomanometric recordings and AHI. Secondary outcome measures were symptoms related to OSA and quality of life assessed by Mini Rhinoconjunctivitis Quality of Life Questionnaire (MiniRQLQ) scores. To investigate the mechanisms explaining the possible association between OSA and nasal resistance, the findings of clinical ORL examination of patients were also included in the analyses.

\section{MATERIALS AND METHODS \\ Patients}

This study is a part of an on-going randomized, clinical trial exploring the effect of lifestyle intervention including weight loss diet on overweight patients with mild OSA. The detailed 
study design has been earlier presented ${ }^{(7)}$. Previously, we have also reported the impact of a significant weight reduction achieved by a 3-month dietary intervention on nasal breathing ${ }^{(6)}$.

The patients were enrolled if they were aged 18 to 65 years, had mild OSA (AHI 5 - 15), were overweight (BMI 28 - 40), and were treated in the clinic of Otorhinolaryngology of Kuopio University hospital during the years 2004 - 2007. Based on previous studies of lifestyle intervention, we agreed that the lifestyle intervention period of 12 months was considered successful if the patients achieved a minimum of $5 \%$ weight reduction (10,11). The details of the study intervention have been described earlier ${ }^{(7)}$. The patients who succeeded in $5 \%$ weight loss were then divided in two groups based on AHI at the 12-month follow-up. The patients, who were able to diminish their AHI under the cut point value of 5 events/h, were considered to be objectively cured from OSA (non-apnoeic group). Patients in the other group had AHI $\geq 5$ events/h (apnoeic group). For further analysis the patients were also divided in smokers and non-smokers. The study flowchart is presented in Figure 1.

\section{Anthropometric measurements}

At the study site, a trained nurse measured height and weight at the baseline and at the 1-year visit. Body mass index (BMI) was calculated as weight $(\mathrm{kg})$ divided by height ${ }^{2}\left(\mathrm{~m}^{2}\right)$

\section{Sleep recordings}

Nocturnal cardio-respiratory monitoring by Embletta ${ }^{\circledR}$ (Embla, Broomfield, CO, USA) at home was conducted in accordance with accepted guidelines for diagnosing OSA at baseline and at 12-month visit ${ }^{(12)}$. The recordings were manually evaluated blindly with respect to clinical status and group by the same, trained physician. Apnoea was defined as a cessation (more than $90 \%$ ) of airflow for more than 10 seconds with oxygen desaturation for $\geq 4 \%$. Hypopnoea was defined as a reduction (more than $30 \%$ ) of airflow for more than 10 seconds with oxygen desaturation for $\geq 4 \%$. The AHI was defined as the number of apnoeas and hypopnoeas per hour, and mild OSA was defined as AHI 5 - 15 events/h. The OSA was considered objectively cured when AHI $<5$ events $/$ h.

\section{Rhinomanometric measurements}

Rhinomanometry was performed at baseline, and 12-month follow-up visit. A NR6-rhinomanometer (GM Instruments Ltd, Glasgow, UK) was used to make the rhinomanometric recordings, whereby total inspiratory nasal resistance was recorded at a radius of 200 Pascal $^{(13)}$. After a 15 minute acclimatization period, four recordings were made: first in the seated position, next in the supine position after lying down for 5 minutes, third 10 minutes after nasal decongestion in the supine position and the fourth measurement after 5 minutes in the seated position. Nasal decongestion was achieved by swabbing the mucosa of the inferior and middle nasal turbinates with a solution containing $20 \mu \mathrm{g} / \mathrm{ml}$ adrenaline and $40 \mathrm{mg} / \mathrm{ml}$ lidocaine.

\section{Symptoms and quality of life measurements}

Epworth Sleepiness Scale (ESS) ${ }^{(14)}$, Snore Outcome Survey (SOS) ${ }^{(15)}$ and Mini Rhinoconjunctivitis Quality of Life Questionnaire (Mini RQLQ) ${ }^{(16)}$ scores were obtained at the baseline and at 12 months. The ESS is an eight item score that evaluates daytime somnolence in eight specific situations. Total ESS score can vary from 0 (best) to 24 (worst) and a total score exceeding 10 points is considered to be abnormal. The SOS is a disease specific outcome score containing eight items to evaluate the duration, severity, frequency and consequences associated with SDB, especially snoring. Scores on the SOS are normalized on a scale ranging from 0 (worst) to 100 (best). The MiniRQLQ has 14 items in five domains: activity limitations, practical problems, nasal symptoms, eye symptoms and other symptoms. In the questionnaire, the subjects are asked to consider how they had experienced the previous week and to respond to each question on a seven-point scale $(0=$ no impairment, 6 = maximum impairment). In the present study, eye symptoms were not assessed leaving the questionnaire with 9 items. Therefore, the range of total miniRQLQ score can vary from 0 (no impairment) to 54 (maximum impairment). Patient's lifestyle habits, such as smoking were also recorded.

\section{Clinical examination}

Nasal polyposis, septal deviation and nasal congestion were evaluated using a three point scale in each variable $(0=$ no polyps/deviation/congestion, $1=$ minor obstruction caused by polyps/deviation/congestion 2 = nasal cavity entirely obstructed by polyps/deviation/congestion). Based on these individual nasal factors the total nasal airway obstruction was evaluated on a seven-point scale $(0=$ no obstruction, $6=$ total obstruction).

\section{Statistical analysis}

To describe the characteristics of two study groups, mean values and standard deviations were used. T-test and Fisher's exact test were used to assess equality between the treatment groups at baseline and at 12-month follow-up. The statistical significance of differences in changes between the groups was assessed with analysis of covariance (ANCOVA), with adjustment for baseline values. A 95 percent confidence interval (CI) was calculated for the main outcome measures. The differences between groups were regarded statistically significant if a 2-sided p-value was less than 0.05. All characteristics and variables were analyzed with the Statistical Package for Social Sciences program (SPSS software, version 17 for Windows; SPSS Inc, Chicago, IL, USA).

\section{RESULTS}

A total of $26 / 52$ patients succeeded in $\geq 5 \%$ weight reduction during the 12 month follow-up, and of those 16 improved their AHI under 5 events/hour, and thus, were considered to be cured from OSA. However, despite the significant weight reduction, the AHI of 10/26 remained at $\geq 5$ events/hour. There was no significant difference in the clinical findings between the apnoeic and non-apnoeic groups i.e. regarding 
nasal polyposis, septal deviation or nasal congestion evaluated by clinical examination both at the baseline and at the 12-month follow-up visit. At baseline, AHI was significantly higher in the apnoeic group compared to the non-apnoeic group, whereas the improvement of AHI at the follow-up was significantly greater in the non-apnoeic group. Furthermore, in the apnoeic group, $40 \%(4 / 10)$ of the patients were cigarette smokers, whereas in the non-apnoeic group 19\% (3/16) of the patients were smokers (Table 1). One patient from the apnoeic group managed to quit smoking during the follow-up, but none succeeded from the apnoeic group.

\section{Rhinometric measurements}

At the 12-month visit, the mean nasal resistance in the supine position in the non-apnoeic group was significantly less $(0.14$
$\left.\mathrm{Pa} / \mathrm{cm}^{3} / \mathrm{s}\right)$ than in patients of the apnoeic group $\left(0.29 \mathrm{~Pa} / \mathrm{cm}^{3} / \mathrm{s}\right.$, $\mathrm{p}=0.006$ ). The mean change in nasal resistance during the 12- month follow-up was $-0.13 \mathrm{~Pa} / \mathrm{cm}^{3} / \mathrm{s}$ in the non-apnoeic group and $0.01 \mathrm{~Pa} / \mathrm{cm}^{3} / \mathrm{s}$ in the apnoeic group $(\mathrm{p}=0.006)$. At 12 months, the effect of nasal decongestion in the supine position was significantly more in the apnoeic group $(-0.13$ $\left.\mathrm{Pa} / \mathrm{cm}^{3} / \mathrm{s}\right)$ compared to the non-apnoeic group $\left(-0.03 \mathrm{~Pa} / \mathrm{cm}^{3} / \mathrm{s}\right.$, $\mathrm{p}=0.02)$. Furthermore, the subjects in the apnoeic group displayed a greater mean change due to nasal decongestion in the follow-up period compared to the non-apnoeic group $(p=0.03)$. (Table 2).

When the 26 patients with a successful weight loss were divided into smokers $(n=7)$ and non-smokers $(n=19)$, the mean change in nasal resistance was $0.06 \mathrm{~Pa} / \mathrm{cm}^{3} / \mathrm{s}$ in smokers and

Table 1. Patient characteristics at baseline and mean changes over the 12-month follow-up.

\begin{tabular}{lccc}
\hline & $\begin{array}{c}\text { Apnoeic group } \\
\mathrm{n}=10(\mathrm{SD})\end{array}$ & $\begin{array}{c}\text { Non-apnoeic group } \\
\mathrm{n}=16(\mathrm{SD})\end{array}$ & $\mathrm{p}$ \\
\hline Age (years) & $52(7.6)$ & $50(7.9)$ & 0.50 \\
Gender (m/f) & $8 / 2$ & $13 / 3$ & 1.0 \\
Weight (kg) & $105(14)$ & $96(12)$ & 0.099 \\
Mean change in weight (kg) & $-11.4(7.4)$ & $-12.9(5.1)$ & 0.55 \\
BMI (kg/m2) & $33(3.6)$ & $32(2.5)$ & 0.43 \\
Mean change in BMI & $-3.7(2.4)$ & $-4.3(1.6)$ & 0.43 \\
AHI (events/hour) & $13(3.2)$ & $9(2.7)$ & 0.003 \\
Mean change in AHI & $-1.2(6.2)$ & $-7.0(2.4)$ & 0.003 \\
Smokers/nonsmokers & $4 / 6$ & $3 / 13$ & 0.37 \\
\hline
\end{tabular}

t-test for continuous variables, Fisher's exact test for dichotomous variables; AHI (apnoea-hypopnea index); BMI (body mass index).

Table 2. Rhinomanometric measurements in supine position.

\begin{tabular}{|c|c|c|c|c|}
\hline & & $\begin{array}{l}\text { Apnoeic group } \\
\text { (SD) }\end{array}$ & $\begin{array}{l}\text { Non-apnoeic group } \\
\text { (SD) }\end{array}$ & $\mathrm{p \#}$ \\
\hline \multirow{3}{*}{$\begin{array}{l}\text { Mean total nasal inspiratory } \\
\text { resistance } \\
(\mathrm{Pa} / \mathrm{cm} 3 / \mathrm{s})\end{array}$} & Baseline & $\begin{array}{c}0.28 \\
(0.15)\end{array}$ & $\begin{array}{c}0.27 \\
(0.12)\end{array}$ & 0.88 \\
\hline & 12 months & $\begin{array}{c}0.29 \\
(0.18)\end{array}$ & $\begin{array}{c}0.14 \\
(0.08)\end{array}$ & 0.006 \\
\hline & Mean Change & $\begin{array}{c}0.01 \\
(0.25)\end{array}$ & $\begin{array}{l}-0.13^{*} \\
(0.20)\end{array}$ & 0.006 \\
\hline \multirow{3}{*}{$\begin{array}{l}\text { Mean total nasal inspiratory } \\
\text { resistance, } \\
\text { decongested }\end{array}$} & Baseline & $\begin{array}{c}0.25 \\
(0.08)\end{array}$ & $\begin{array}{c}0.22 \\
(0.05)\end{array}$ & 0.51 \\
\hline & 12 months & $\begin{array}{c}0.17 \\
(0.09)\end{array}$ & $\begin{array}{c}0.12 \\
(0.06)\end{array}$ & 0.07 \\
\hline & Mean Change & $\begin{array}{l}-0.08^{*} \\
(0.11)\end{array}$ & $\begin{array}{l}-0.11 * * \\
(0.11)\end{array}$ & 0.51 \\
\hline \multirow{3}{*}{ Effect of nasal decongestion } & Baseline & $\begin{array}{l}-0.03 \\
(0.14)\end{array}$ & $\begin{array}{l}-0.05 \\
(0.18)\end{array}$ & 0.52 \\
\hline & 12 months & $\begin{array}{l}-0.13 \\
(0.14)\end{array}$ & $\begin{array}{l}-0.03 \\
(0.07)\end{array}$ & 0.02 \\
\hline & Mean Change & $\begin{array}{l}-0.10 \\
(0.20)\end{array}$ & $\begin{array}{c}0.02 \\
(0.18)\end{array}$ & 0.03 \\
\hline
\end{tabular}

Asterisks indicate whether the change between follow-up and baseline was significant in the respective group: ${ }^{*} \mathrm{p}<0.05,{ }^{* *} \mathrm{p}<0.01$ and ${ }^{* * *} \mathrm{p}<0.001$; paired-samples t-test. ${ }^{*}$ Indepent-samples t-test, or ANCOVA for changes, adjusted for baseline. 


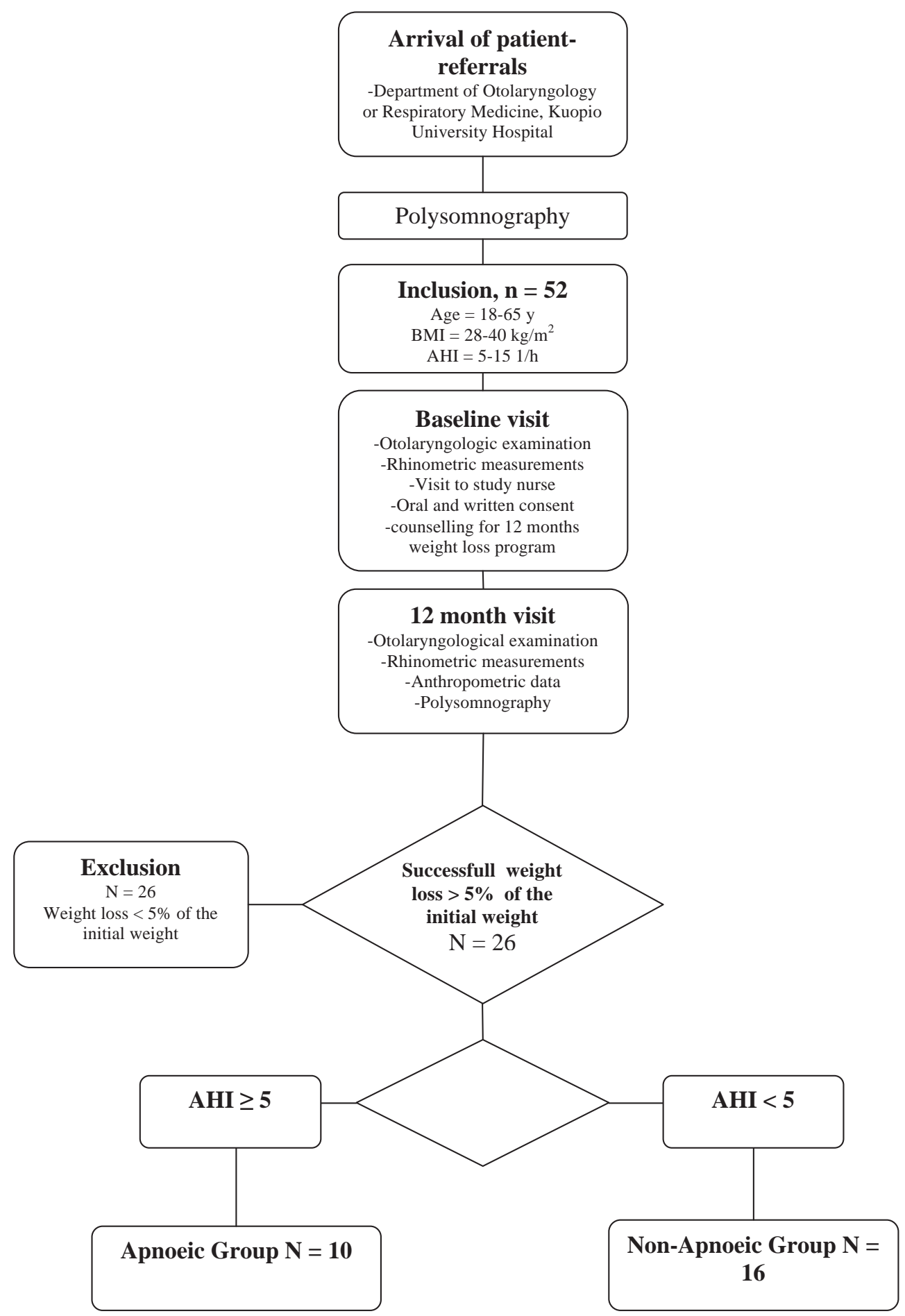

Figure 1. Study flowchart.

$-0.12 \mathrm{~Pa} / \mathrm{cm}^{3} / \mathrm{s}$ in non-smokers $(\mathrm{p}=0.009)$. Furthermore, the mean change in AHI in cigarette smokers compared with nonsmokers was -1.4 and -6.0 , respectively $(p=0.035)$.

In the correlation between the change in BMI and the change in nasal resistance was a trend to significance with the current sample size (Pearson correlation coefficient $r=0.262$, $\mathrm{p}=0.07)$.
Symptom scores and quality of life measures

The decrease of symptoms according to ESS was 4.4 pts in the non-apnoeic group, which was significantly more when compared with a decrease of $0.6 \mathrm{pts}$ in the apnoeic group ( $\mathrm{p}$ $=0.037$ ). The total ESS score at twelve months was 5.8 in the non-apnoeic group and 8.7 in the apnoeic group, but the difference between the groups was not statistically significant ( $p$ $=0.16$ ). 
The change is SOS score indicated benefit from the intervention in both groups (22.7 in the non-apnoeic group and 6.2 in the apnoeic group), but the change between the groups was not statistically significant $(p=0.077)$. At 12 months total SOS score was 71.2 in the non-apnoeic group and 62.1 in the apnoeic group $(\mathrm{p}=0.14)$.

In MiniRQLQ, both groups reported similar mild impairment in QOL and nose related symptoms. The total miniRQLQ score was 15.0 in the non-apnoeic group and 19.3 in the apnoeic group at the 12-month visit. There was no significant change between the baseline and 12 month measurements in miniRQLQ.

\section{DISCUSSION}

The present study suggests that impaired nasal breathing might be one factor preventing the beneficial effect of weight loss in overweight patients with mild OSA. At the 12-month follow-up, those patients still having OSA despite a successful lifestyle intervention and weight loss had a significantly higher nasal resistance compared with their counterparts who were objectively cured from OSA. Furthermore, patients with OSA had more profound effect of nasal decongestion.

Previously smoking has been found to have adverse effects on the nasal airway. It has been associated with several symptoms i.e. with increased snoring, more nasal obstruction, and pharyngeal soft tissue volume. Overall, the combination of current smoking and altered nasal function seems to compromise the lower airways and thus, predispose to sleep disordered breathing ${ }^{(17,18)}$. The effects of cigarette smoking on the nasal airway remain largely unknown, but they might possibly be secondary to mucosal inflammation ${ }^{(19)}$. In our study, this adverse effect of cigarette smoking on nasal breathing was also observed. In non-smokers, nasal resistance improved during the lifestyle intervention, whereas it tended to increase in smokers. Furthermore, in non-smokers the improvement of AHI was significantly greater compared with that achieved in smokers. Since there were more smokers in the apnoeic-group (40\%) compared with the non-apnoeic group $(19 \%)$, it is possible that cigarette smoking partly prevented the beneficial effects of weight reduction on OSA.

In the present study, no correlation between AHI and nasal resistance was found in either of the groups. The previous data on the effect of nasal breathing on OSA is conflicting. A correlation between nasal resistance and OSA has been found in some studies ${ }^{(4,20)}$, whereas some studies have shown that nasal resistance is not related to the severity of OSA (21) and nasal resistance does not correlate with the AHI ${ }^{(22)}$. Furthermore, the findings on the correlation between nasal breathing and OSA in different weight groups have been controversial. Significant correlations between AHI and nasal resistance have been found in obese patients ${ }^{(23)}$, while in another study this was observed only in non-obese patients ${ }^{(24)}$.
Weight reduction is an effective treatment for OSA and weight loss is recommended in all clinical guidelines for treating OSA when related to obesity ${ }^{(7-9)}$. In our study, correlations between BMI and AHI were significant in all study participants and all patients improved their AHI, although not all of them were cured from OSA. Also, the quality of life and symptoms seemed to be better at the follow-up in the non-apnoeic group compared with the apnoeic group, although the difference was not statistically significant.

There have been only few studies exploring the relationship of obesity and nasal resistance. A correlation between subjective feeling of nasal blockage and obesity was observed in a population based study ${ }^{(5)}$. On the other hand, in a study on young healthy adults, BMI was found not to have effect on nasal resistance ${ }^{(25)}$. Also, our previous study evaluating the effects of intensive weight reduction on rhinomanometric measures did not show any correlation between changes in BMI and nasal resistance ${ }^{(6)}$. In the present study, only a borderline correlation between BMI and rhinomanometric measurements was found. However, this is most likely due to small sample size in this study, as the correlation coefficient was reasonably high.

The main limitation of our study was the considerably small study population and the patients had mild OSA. Thus, these results may not be generalized to all OSA patients. At baseline, there was a small but statistically significant difference in AHI between the groups.

In conclusion, impaired nasal breathing may reduce the beneficial effects of weight reduction in the treatment of overweight patients with OSA. Therefore, the assessment of nasal breathing and educational guidance for smoking should always be included in the management of OSA patients.

\section{ACKNOWLEDGEMENTS}

The members of Kuopio Sleep Apnoea Group Taina Poutiainen, Matti Pukkila, Grigori Smirnov, Tatu Kemppainen, Tomi Laitinen, Tiina Lyyra-Laitinen, Aki Ikonen, Ritva Vanninen, Heimo Viinamäki, Keijo Peuhkurinen, Kari Punnonen, Kati Venäläinen, Riitta Pahkala, Erkki Soini and Janne Martikainen are cordially acknowledged.

\section{AUTHORSHIP CONTRIBUTION}

HT and JSE were responsible for the general conception and study design. HB drafted the manuscript, with input from TK, JSE, JSA, JN, PR and HT. HT coordinated the study, MP and HB performed the statistical analyses. JSA, PR, TK, JSE, HT and HB contributed to clinical work related to the study and participated in interpreting the results and in editing of the manuscript. All authors have approved the current version.

\section{CONFLICT OF INTEREST}

None. 


\section{REFERENCES}

1. Young T, Peppard PE, Gottlieb DJ. Epidemiology of obstructive sleep apnea: A population health perspective. Am J Respir Crit Care Med. 2002; 165: 1217-1239.

2. Punjabi NM, Caffo BS, Goodwin JL, et al. Sleep-disordered breathing and mortality: A prospective cohort study. PLoS Med. 2009; 6: e1000132.

3. Young T, Skatrud J, Peppard PE. Risk factors for obstructive sleep apnea in adults. JAMA. 2004; 291: 2013-2016.

4. Lofaso F, Coste A, d' Ortho MP, et al. Nasal obstruction as a risk factor for sleep apnoea syndrome. Eur Respir J. 2000; 16: 639-643.

5. Johansson L, Bende M. Excessive obesity is related to daily symptoms of nasal blockage: The skovde population-based study. Rhinology 2007; 45:205-207.

6. Kemppainen T, Ruoppi P, Seppa J, et al. Effect of weight reduction on rhinometric measurements in overweight patients with obstructive sleep apnea. Am J Rhinol. 2008; 22: 410-415.

7. Tuomilehto HP, Seppa JM, Partinen MM, et al. Lifestyle intervention with weight reduction: First-line treatment in mild obstructive sleep apnea. Am J Respir Crit Care Med. 2009; 179: 320-327.

8. Foster GD, Borradaile KE, Sanders MH, et al. A randomized study on the effect of weight loss on obstructive sleep apnea among obese patients with type 2 diabetes: The sleep AHEAD study. Arch Intern Med. 2009; 169: 1619-1626.

9. Johansson K, Neovius M, Lagerros YT, et al. Effect of a very low energy diet on moderate and severe obstructive sleep apnoea in obese men: A randomised controlled trial. BMJ. 2009; 339: b4609.

10. Lindstrom J, Peltonen M, Eriksson JG, et al. Determinants for the effectiveness of lifestyle intervention in the finnish diabetes prevention study. Diabetes Care. 2008; 31: 857-862.

11. Uusitupa M, Peltonen M, Lindstrom J, et al. Ten-year mortality and cardiovascular morbidity in the finnish diabetes prevention study--secondary analysis of the randomized trial. PLoS One. 2009; 4: e5656.

12. Anonymous. Sleep-related breathing disorders in adults: Recommendations for syndrome definition and measurement techniques in clinical research. the report of an american academy of sleep medicine task force. Sleep. 1999; 22: 667-689.

13. Broms P, Jonson B, Lamm CJ. Rhinomanometry. II. A system for numerical description of nasal airway resistance. Acta Otolaryngol. 1982; 94: 157-168.

14. Johns MW. A new method for measuring daytime sleepiness: The epworth sleepiness scale. Sleep. 1991; 14: 540-545.

15. Gliklich RE, Wang PC. Validation of the snore outcomes survey for patients with sleep-disordered breathing. Arch Otolaryngol Head Neck Surg. 2002; 128: 819-824.
16. Juniper EF, Thompson AK, Ferrie PJ, Roberts JN. Development and validation of the mini rhinoconjunctivitis quality of life questionnaire. Clin Exp Allergy. 2000; 30: 132-140.

17. Virkkula P, Hytonen M, Bachour A, et al. Smoking and improvement after nasal surgery in snoring men. Am J Rhinol. 2007; 21: 169-173.

18. Virkkula P, Bachour A, Hytonen M, Malmberg H, Salmi T, Maasilta P. Patient- and bed partner-reported symptoms, smoking, and nasal resistance in sleep-disordered breathing. Chest. 2005; 128: 2176-2182.

19. Kjaergaard T, Cvancarova M, Steinsvaag SK. Smoker's nose: Structural and functional characteristics. Laryngoscope. 2010; 120: 1475-1480.

20. Li HY, Wang PC, Hsu CY, Cheng ML, Liou CC, Chen NH. Nasal resistance in patients with obstructive sleep apnea. ORL J Otorhinolaryngol Relat Spec. 2005; 67: 70-74.

21. Miljeteig H, Hoffstein V, Cole P. The effect of unilateral and bilateral nasal obstruction on snoring and sleep apnea. Laryngoscope. 1992; 102: 1150-1152.

22. Yagi H, Nakata S, Tsuge H, et al. Morphological examination of upper airway in obstructive sleep apnea. Auris Nasus Larynx. 2009; 36: 444-449.

23. Tagaya M, Nakata S, Yasuma F, et al. Pathogenetic role of increased nasal resistance in obese patients with obstructive sleep apnea syndrome. Am J Rhinol Allergy. 2010; 24: 51-54.

24. Virkkula P, Maasilta P, Hytonen M, Salmi T, Malmberg H. Nasal obstruction and sleep-disordered breathing: The effect of supine body position on nasal measurements in snorers. Acta Otolaryngol. 2003; 123: 648-654.

25. Numminen J, Ahtinen M, 3rd, Huhtala H, Laranne J, Rautiainen M. Correlation between rhinometric measurement methods in healthy young adults. Am J Rhinol. 2002; 16: 203-208.

Henry Blomster, on the behalf of all the authors

Department of Otorhinolaryngology

Kuopio University Hospital

P.O. Box 1777

FIN-70211 Kuopio

Finland

Fax: 358-17-172509

Tel: 358-17-173311

E-mail: henry.blomster@kuh.fi 